\title{
Análisis bibliométrico de las revistas de enfermedades respiratorias de América Latina ${ }^{\#}$
}

\author{
MANUEL OYARZÚN G.* y ROSSANA ZÚÑIGA B.**
}

\section{A bibliometric analysis of Latin American journals on respiratory diseases}

In 2007, the editorial situation of seven Latin American journals on respiratory diseases was analyzed in a meeting sponsored by ALAT (Latin American Association of Thoracic Diseases). Among the major problems reported were the lack of a regular and adequate periodicity in issue's circulation, high turnover of editors and lack of original papers submitted, that lead to a low percentage of submitted papers rejection. The present situation of Latin American journals on respiratory diseases is currently compared with the Iberian Peninsula journals Archivos de Bronconeumología and Revista Portuguesa de Pneumologia, through the international data baseLILACS (Literatura Latinoamericana en Ciencias de la Salud), SciELO (Scientific Electronic Library on Line), SCImago Journal \& Country Rank, MEDLINE (National Library of Medicine, USA) and JCR (Journal Citation Report). The Jornal Brasileiro de Pneumologia is the Latin American journal with the best bibliometric indices; nevertheless, it is ranked behind Spain's Archivos de Bronconeumologia (ABN). Interestingly 11\% of the 238 citable articles published in ABN from 2013 to 2015 had authors from Latin America. Conclusions: Although Latin American journals on respiratory diseases disseminate valuable information in their national environment accomplishing an educational role, their global impact and bibliometric indices are exiguous.

Key words: Bibliometrics; thoracic diseases; Latin America; Spain; publication.

\section{Resumen}

En una reunión patrocinada por la ALAT (Asociación Latinoamericana de Tórax) realizada en 2007, se analizó la situación editorial de siete revistas latinoamericanas de enfermedades respiratorias. Entre los principales problemas se señalaron la carencia de una periodicidad regular y adecuada en la publicación de los fascículos, el elevado recambio de editores y la escasez de manuscritos originales enviados para su eventual publicación, de lo cual resulta un bajo porcentaje de rechazo en los manuscritos sometidos a revisión. La actual situación de las revistas latinoamericanas de enfermedades respiratorias es comparada en este artículo con las revistas de la península ibérica Archivos de Bronconeumología y Revista Portuguesa de Pneumologia, mediante las bases de datos internacionales LILACS (Literatura Latinoamericana en Ciencias de la Salud), SciELO (Scientific Electronic Library on line), SCImago Journal \& Country Rank, MEDLINE (National Library of Medicine, USA) y JCR (Journal Citation Report). El Jornal Brasileiro de Pneumologia es la revista latinoamericana que tiene los mejores índices bibliométricos, sin embargo, está clasificada por debajo de Archivos de Bronconeumología (ABN). Es interesante destacar que $11 \%$ de los 238 artículos citables publicados en ABN entre 2013 y 2015 fueron generados por autores de América Latina. Conclusiones: Si bien las revistas latinoamericanas de enfermedades respiratorias difunden una valiosa información en sus ambientes nacionales cumpliendo una función educacional, tanto su impacto global como sus índices bibliométricos son exiguos.

Palabras clave: Bibliometría; enfermedades torácicas; América Latina; España; publicaciones.

* Instituto de Ciencias Biomédicas, Facultad de Medicina, Universidad de Chile y Editor, Revista Chilena de Enfermedades Respiratorias.

** Bibliotecaria, Biblioteca Médica Prof. Dr. Hernán Alessandri, Campus Oriente, Facultad de Medicina, Universidad de Chile.

\# Texto basado en la conferencia pronunciada el Simposio: "Presente y futuro de las revistas respiratorias en América Latina" en el $10^{\circ}$ Congreso de la Asociación Latinoamericana de Tórax (ALAT), Santiago de Chile, 7 de Julio de 2016. 


\section{Introducción}

En 2005 un análisis bibliométrico de la producción global de publicaciones sobre investigaciones en enfermedades respiratorias ubicó a América Latina en la penúltima posición entre las 9 regiones analizadas ${ }^{1}$. La contribución de América Latina a un total de 48.614 publicaciones ISI (Institute for Scientific Information) referidas por PubMed entre 1995 y 2003 fue de tan sólo 1.250, es decir 1\% (Figura 1). En ese mismo estudio, el número de publicaciones sobre investigaciones de todo el mundo en enfermedades respiratorias separadas en 9 regiones se ajustó por ingreso nacional bruto per cápita y se expresó por millón de habitantes. El resultado fue aún más dramático, ya que América Latina quedó ubicada en el último lugar de ese ranking (Figura 2).

La situación de las revistas de enfermedades respiratorias publicadas en América Latina, fue analizada hace ya más de 9 años por los editores de siete de estas revistas en una reunión organizada por ALAT (Asociación Latinoamericana de Tórax) el 4 de febrero de 2007 en Lima, Perú 2.

Concurrieron a esta reunión los editores y/o representantes de las revistas de enfermedades respiratorias patrocinadas por las sociedades nacionales de la especialidad de Argentina, Brasil, Chile, Colombia, México, Perú y la Federación Centroamericana y del Caribe.

Estas siete revistas de enfermedades respiratorias representaban a la gran mayoría de las
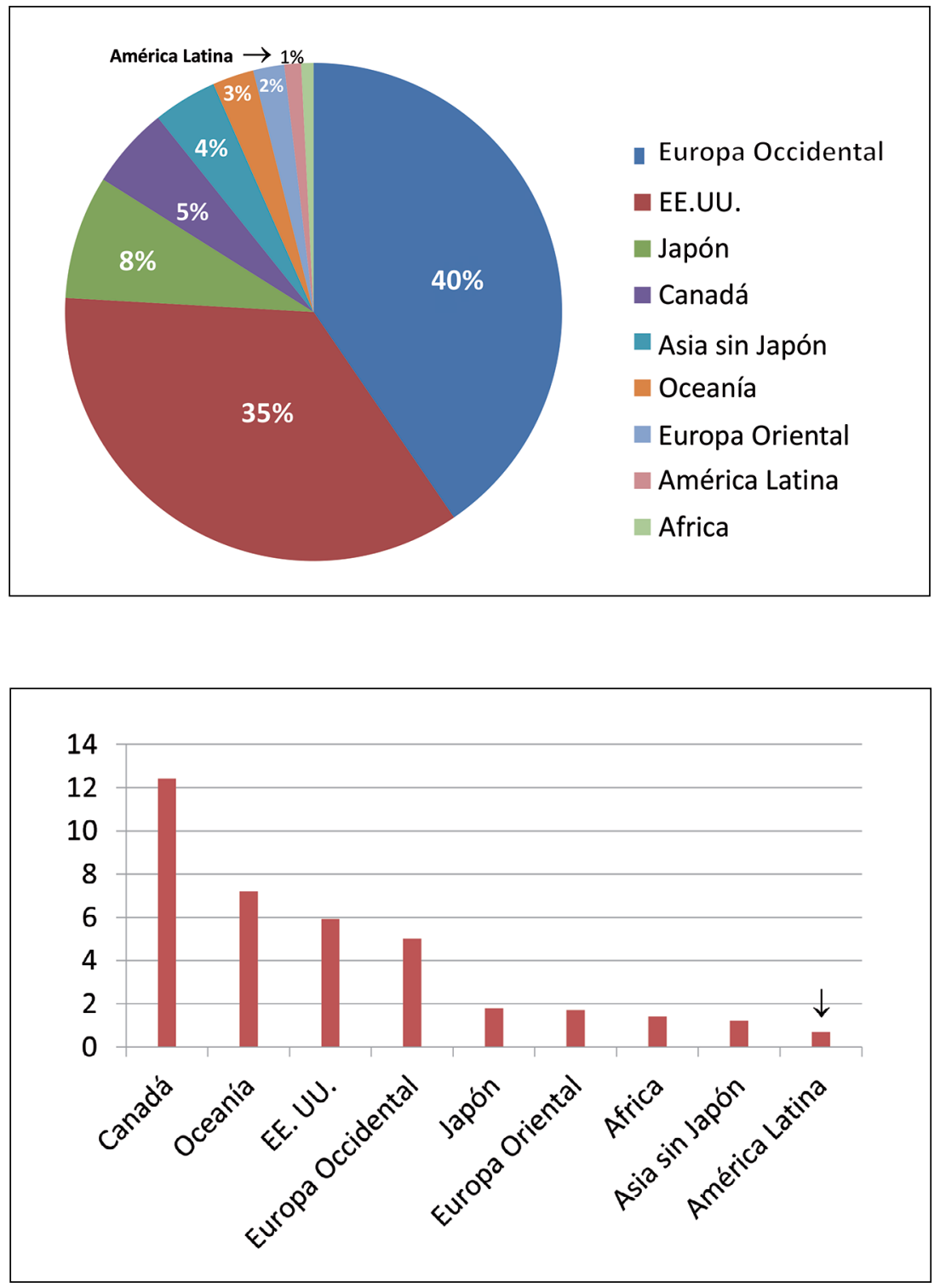

Figura 1. Distribución porcentual de un total de 48.614 artículos sobre sistema respiratorio publicados en revistas JCR (Journal Citation Report) de diferentes regiones del mundo entre 1995 y 2003. Gráfico elaborado con cifras de Michalopoulus y Falaga ${ }^{1}$.

Figura 2. Número de publicaciones por millón de habitantes sobre sistema respiratorio entre 1995 y 2003 en revistas ISI ajustadas por ingreso nacional bruto per cápita (GINPC). Gráfico elaborado con cifras de Michalopoulus y Falaga ${ }^{1}$. 
revistas de esta especialidad que se publicaban en América Latina. En esta región en el año 2007 existían 9 revistas de enfermedades respiratorias, según búsqueda realizada en 2006 por el Dr. Rogelio Pérez-Padilla, presidente de ALAT en esa época y quien convocó a esta reunión ${ }^{3}$. En la reunión de Lima no estuvieron representadas la Revista del Instituto Nacional de Enfermedades Respiratorias de México editada por ese Instituto y Archivos de Bronconeumología, patrocinada por la SEPAR (Sociedad Española de Neumología y Cirugía Torácica) y ALAT. Sin embargo, se estimó que los informes de los representantes de las siete revistas concurrentes, constituían una muestra representativa de lo que ocurría con la edición de revistas de enfermedades respiratorias, que son órganos oficiales de sociedades nacionales y regionales de enfermedades respiratorias en América Latina ${ }^{4}$.

Cuatro de estas siete revistas destinaban $30 \%$ o más de su espacio a publicar trabajos originales, los cuales fluctúan entre 3 a 12 artículos por número. Las restantes publicaban mayoritariamente artículos de revisión, casos clínicos y resúmenes de congresos ${ }^{2}$.

En relación al financiamiento, seis de las siete revistas eran financiadas por avisaje de la industria farmacéutica y en proporciones variables por la sociedad médica que representan y su distribución en general era gratuita. El Jornal Brasileiro de Pneumologia recibió además, un subsidio de investigación del Estado de Brasil que financió un $5 \%$ de los gastos de publicación ${ }^{2}$.

Los principales problemas detectados por los editores de estas revistas en la reunión de Lima fueron los siguientes ${ }^{2,4}$ :

1. Carencia de continuidad y periodicidad regular de su publicación. Lo mínimo es lograr una periodicidad trimestral de números regulares, excluyéndose los consensos y comunicaciones libres a congresos, los que deberían ser publicados como un número suplemento.

2. Escasez de suficientes artículos originales que signifiquen un real impacto en la especialidad. Deberían publicarse 2 o 3 de estos artículos por año, si se desea que la revista sea aceptada en índices internacionales exigentes. Los artículos reescritos de originales publicados por los mismos autores en otras revistas o artículos de revisión, especialmente cuando son escritos por personas sin experiencia en el tema, no tienen mayor relevancia.

3. En general, el porcentaje de rechazo de trabajos originales es bajo: 5\%-39\% comparado con el rechazo de artículos en revistas de alto impacto $(80 \%)$, lo que indicaría una limitación en la capacidad para seleccionar artículos de calidad competitiva y universal en la mayoría de estas revistas. La mayor parte de las revistas registró una elevada rotación de editores, habitualmente cada dos años, lo cual dificulta una adecuada gestión y cumplimiento de normas internacionales de edición. Lo expuesto en este punto, podría explicar el escaso número de revistas latinoamericanas de neumología incluidas en índices internacionales de mayor exigencia.

Las estrategias más usadas por los editores para mejorar la calidad de los manuscritos fueron tres: a) mejorar la relación entre autor-editorrevisor; b) mejorar la distribución y difusión de la revista y c) garantizar la periodicidad de la publicación.

Las sugerencias de los editores acerca de cómo mejorar las revistas Latinoamericanas de Enfermedades Respiratorias, se resumen en profesionalizar el trabajo editorial, aumentar el grado de meticulosidad de los revisores de los manuscritos y seguir rigurosamente las normas internacionales para editar revistas ${ }^{4}$.

A pesar de la elevada prevalencia de las enfermedades respiratorias en el mundo, las revistas en salud respiratoria que se publicaban en América Latina en 2007 no reflejaban la importancia que tienen las enfermedades respiratorias. Con el propósito de mancomunar esfuerzos y proponer opciones de lograr un medio de comunicación más eficaz, la reunión de editores de Lima del año 2007 acordó crear una comisión multinacional para preparar un anteproyecto de revista latinoamericana, que explorara la factibilidad de lograr este propósito en la ALAT, acción que no se logró concretar.

\section{¿Cuál es la situación actual de las revistas de enfermedades respiratorias en América Latina?}

Para contestar esta pregunta revisamos las revistas de América Latina que están incluidas en las bases de datos de indexación internacional LILACS (Literatura Latinoamericana en Ciencias de la Salud) ${ }^{5}$, SciELO (Scientific Electronic Library on line $)^{6}$, SCImago Journal \& Country Rank $^{7}$, MEDLINE (National Library of Medicine, USA $)^{8}$ y JCR (Journal Citation Report, revistas ISI-Thomas $)^{9}$ y las comparamos con la indexación de las dos principales revistas de enfermedades respiratorias de España y Portugal, Archivos de Bronconeumología y Revista Portuguesa de 
Pneumologia respectivamente (Tabla 1). Se pudo constatar que la única revista latinoamericana que ha logrado ser incluida en todos estos índices es el Jornal Brasileiro de Pneumologia. Sin embargo, su factor de impacto JCR (Journal Citation Report) aún está por debajo de sus revistas pares de la península ibérica. La Revista Portuguesa de Pneumologia ha estado aplicando una estrategia editorial ${ }^{10}$ que le ha permitido aumentar paulatinamente su factor de impacto JCR a valores por sobre 1. Es un hecho conocido que la mejor manera de aumentar el factor de impacto JCR de una revista es publicar artículos de la más alta calidad, sin embargo es difícil atraer manuscritos de gran calidad para que sean publicados en revistas con bajo factor de impacto. Para mejorar el factor de impacto en esas circunstancias es necesario que los comités editoriales comprendan el proceso de evaluación de las revistas y las estrategias que se pueden adoptar legítimamente para incrementar su valor ${ }^{11}$. Entre las intervenciones que las revistas pueden adoptar para mejorar su situación en la evaluaciones bibliométricas se han señalado las siguientes ${ }^{11}$ : Invitar a publicar en la revista a autores líderes en determinados temas, publicar artículos especialmente relevantes en el primer número del año, lo cual les da un mayor tiempo para ser citados, dar preferencia a los artículos originales y a las revisiones, promover la divulgación de los mejores artículos en los medios sociales, para aumentar la visibilidad de la revista, mejorar la calidad del proceso de revisión por pares, publicar editoriales interesantes y atractivos que estimulen a leer la revista, evitar demoras injustificadas en las revisiones y decisiones de publicación, mantener una razonable estabilidad del cuerpo editorial y si es posible estimular que los artículos aceptados sean publicados en línea previos a ser impresos ("ahead on print/on line first").

Otro hecho interesante que ha ocurrido en estos 9 años post reunión de editores en Lima 2007 es que las sociedades nacionales de Argentina y México han hecho un esfuerzo por incorporar a su revista el patrocinio de otras sociedades nacionales neumológicas de América Latina (Tabla 2), como esta iniciativa es reciente, aún es muy temprano para objetivar su impacto en sus índices bibliométricos. Por otra parte, en Chile se ha incorporado desde 2009 a LILACS una nueva revista dedicada exclusivamente a enfermedades respiratorias pediátricas: Revista de Neumología Pediátrica (ex Boletín de Neumología Pediátrica) es órgano oficial Sociedad Chilena de Neumo-

Tabla 1. Revistas latinoamericanas y de la península ibérica indexadas en base de datos internacionales

\begin{tabular}{|c|c|c|c|c|c|c|}
\hline \multirow[t]{2}{*}{ País } & \multirow[t]{2}{*}{ Revista/Editor(a) } & \multicolumn{5}{|c|}{ Base de datos indexada } \\
\hline & & LILACS & SciELO & SCImago & MEDLINE & JCR \\
\hline Argentina & $\begin{array}{l}\text { Revista Americana de Medicina Respiratoria* } \\
\text { Dra. Silvia Quadrelli }\end{array}$ & $\diamond$ & $\diamond$ & & & \\
\hline Brasil & $\begin{array}{l}\text { Jornal Brasileiro de Pneumología } \\
\text { Dr. Rogerio Souza }\end{array}$ & $\diamond$ & $\diamond$ & $\diamond$ & $\bullet$ & $\stackrel{\overrightarrow{1}}{\bullet}$ \\
\hline \multirow[t]{2}{*}{ Chile } & $\begin{array}{l}\text { Revista Chilena de Enfermedades Respiratorias } \\
\text { Dr. Manuel Oyarzún Gómez }\end{array}$ & $\diamond$ & $\bullet$ & $\diamond$ & & \\
\hline & $\begin{array}{l}\text { Revista de Neumología Pediátrica } \\
\text { Dra. Solange Caussade Larraín }\end{array}$ & $\diamond$ & & & & \\
\hline Colombia & $\begin{array}{l}\text { Revista Colombiana de Neumología } \\
\text { Dr. Carlos Andrés Celis Preciado }\end{array}$ & $\diamond$ & & & & \\
\hline \multirow[t]{2}{*}{ México } & $\begin{array}{l}\text { Revista del Instituto Nacional de Enfermedades } \\
\text { Respiratorias } \\
\text { Dra. Ma. E. Yuriko Furuya Meguro }\end{array}$ & $\diamond$ & $\diamond$ & $\diamond$ & & \\
\hline & $\begin{array}{l}\text { Neumología y Cirugía de Tórax* } \\
\text { Dr. Patricio Santillán Doherty }\end{array}$ & & $\diamond$ & & & \\
\hline Portugal & $\begin{array}{l}\text { Revista Portuguesa de Pneumología } \\
\text { Dr. António Morais }\end{array}$ & $\mathrm{Li}^{\#}$ & $\bullet$ & $\bullet$ & $\diamond$ & $\stackrel{\bullet}{1,167^{+}}$ \\
\hline España & $\begin{array}{l}\text { Archivos de Bronconeumología } \\
\text { Dra. Esther Barreiro }\end{array}$ & $\mathrm{Li}^{\#}$ & $\diamond$ & $\bullet$ & $\diamond$ & $\stackrel{\bullet}{1,771^{+}}$ \\
\hline
\end{tabular}

*Órganos oficiales de la sociedad del país de origen y de otras sociedades neumológicas de América Latina. ${ }^{\#} \mathrm{Li}=\mathrm{La}-$ tindex + Factor de impacto JCR (Journal Citation Report). 
Tabla 2. Revistas latinoamericanas de enfermedades respiratorias indexadas en SciELO

\begin{tabular}{|c|c|c|c|c|c|c|}
\hline País & Revista & Patrocinador & Año $0^{\#}$ & Vol. & Periodicidad & $\begin{array}{l}\text { Factor de } \\
\text { impacto* }\end{array}$ \\
\hline Argentina & $\begin{array}{l}\text { Revista Americana } \\
\text { de Medicina } \\
\text { Respiratoria Antes: } \\
\text { Revista Argentina de } \\
\text { Medicina Respiratoria }\end{array}$ & $\begin{array}{l}\text { Asociación Argentina de } \\
\text { Medicina Respiratoria } \\
\text { Sociedades Boliviana, } \\
\text { Ecuatoriana, Paraguaya, } \\
\text { Uruguaya y Venezolana } \\
\text { de Neumologia (y CT) }\end{array}$ & $\begin{array}{l}\text { 2011-a la } \\
\text { actualidad }\end{array}$ & 15 & Trimestral & 0,1026 \\
\hline Brasil & $\begin{array}{l}\text { Jornal Brasileiro de } \\
\text { Pneumologia. } \\
\text { Antes: Jornal de } \\
\text { Pneumologia }\end{array}$ & $\begin{array}{l}\text { Sociedade Brasileira de } \\
\text { Pneumologia e Tisiologia }\end{array}$ & $\begin{array}{l}\text { 2004-a la } \\
\text { actualidad }\end{array}$ & 42 & Bimestral & 0,3220 \\
\hline Chile & $\begin{array}{l}\text { Revista Chilena de } \\
\text { Enfermedades } \\
\text { Respiratorias } \\
\text { Antes: Enfermedades } \\
\text { Respiratorias y Cirugía } \\
\text { Torácica }\end{array}$ & $\begin{array}{l}\text { Sociedad Chilena de } \\
\text { Enfermedades } \\
\text { Respiratorias }\end{array}$ & $\begin{array}{l}\text { 2002-a la } \\
\text { actualidad }\end{array}$ & 31 & Trimestral & 0,3333 \\
\hline \multirow[t]{2}{*}{ México } & $\begin{array}{l}\text { Revista del Instituto } \\
\text { Nacional de Enfermedades } \\
\text { Respiratorias }\end{array}$ & $\begin{array}{l}\text { Instituto Nacional } \\
\text { de Enfermedades } \\
\text { Respiratorias: INER }\end{array}$ & 2004-2007 & 20 & Trimestral & \multirow[t]{2}{*}{0,0286} \\
\hline & $\begin{array}{l}\text { Neumología y Cirugía } \\
\text { de Tórax }\end{array}$ & $\begin{array}{l}\text { Sociedad Mexicana de } \\
\text { Neumología y Cirugía de } \\
\text { Tórax; INER+ Sociedades } \\
\text { Boliviana, Cubana y } \\
\text { Paraguaya de Neumología } \\
\text { y Fed. Centroamericana y } \\
\text { del Caribe N y CT }\end{array}$ & 2014-2015 & 74 & Trimestral & \\
\hline
\end{tabular}

*Factor de impacto: medición de la frecuencia con la que el "artículo promedio" de una revista se ha citado en un año en la colección SciELO. CT: cirugía torácica. N: neumología. "Año: ingreso a SciELO.

logía Pediátrica (SOCHINEP) y de la Sociedad Latinoamericana de Neumología (SOLANEP) publicando 4 ediciones anuales (Tabla 1). Esta revista al igual que la Revista Colombiana de Neumología no se ha incorporado aun a otras bases de datos de mayor exigencia por lo que ambas no se incluyeron en el ulterior análisis bibliométrico.

Al efectuar una revisión en la Base de Datos Scientific Electronic Library Online (SciELO) ${ }^{6}$, para identificar a los países de América Latina que publican revistas de enfermedades respiratorias, constatamos que Argentina, Brasil, Chile y México tienen revistas indexadas en este tema, con una periodicidad bi o trimestral (Tabla 2).

También revisamos la base de datos en bibliometría SCImago Journal \& Country Rank ${ }^{7}$, creada por un grupo de estudio de España y dedicada al análisis de información y al desarrollo de herramientas de ranking. SCImago Journal \& Country Rank obtiene esta información de las revistas indexadas y con visualización en la base de datos Scopus.

Los indicadores principales de la base de datos en la bibliometría Scimago son el SJR del SCImago Journal \& Country Rank (Portal de indicadores bibliométricos basados en citas, de la base de datos Scopus de Elsevier Publishing Co.) y el índice "H"7,12.

El SJR (SCImago Journal Rank), es un indicador que expresa el número de contactos que una revista recibe a través de la citación de sus documentos, desglosados de entre el total de documentos publicados en el año seleccionado por la publicación, ponderados según la cantidad de contactos o citas que están entrando y saliendo de las fuentes ${ }^{7,12}$.

El índice "H" (" $H$ index") de la revista en esta base de datos, cuantifica la productividad científica y el impacto científico de una publicación periódica (también es aplicable para comparar la productividad de investigadores, regiones y países entre otras posibilidades comparativas). Este índice está basado en el conjunto de artículos más citados de la revista y en el número de citas que estos artículos han recibido en otras publicaciones $^{7,12}$. 
Al analizar los países de América Latina que tienen participación en SCImago Journal \& Country Rank con revistas de enfermedades respiratorias encontramos que Brasil, Chile y México tienen indexadas una revista cada uno ${ }^{7}$. Por su parte los dos países de la península ibérica, España y Portugal, tienen revistas en esta área indexadas en esta base de datos ${ }^{7}$ (Tabla 3). Las revistas previamente citadas han sido clasificadas en la Tabla 3 según su índice SJR en orden decreciente, el mayor índice SJR lo obtuvo Archivos de Bronconeumología $(0,432)$ seguido por el Jornal Brasileiro de Pneumologia (0,383). Estos valores están muy por debajo de la revista de enfermedades respiratorias que tiene el más alto índice SJR que es American Journal of Respiratory and Critical Care Medicine (AJRCCM) que alcanza un valor de 5,832. Una situación similar ocurre con el índice $\mathrm{H}$ que en AJRCCM alcanza un valor de 303 superando ampliamente a los valores de las revistas analizadas en la Tabla 3. El número de citas concedidas representa el número de veces que la revista cita a otras revistas o a ella misma, a través de las referencias en sus artículos. Por su parte el número de citas recibidas, se refiere al número de veces que la revista ha sido citada por otras revistas indizadas en la base de datos o por ella misma.

Los instrumentos de la base de datos SCImago Journal \& Country Rank permiten comparar revistas de una misma región entre sí y también las revistas de dos zonas geográficas. Utilizando estos instrumentos de SCImago Journal \& Country $\operatorname{Rank}^{7}$ comparamos el número de artículos citables y la sumatoria de los índices $\mathrm{H}$ del grupo de revistas latinoamericanas de enfermedades respiratorias indexadas en el período 1996-2014 (Figuras 3 y 4). Las tres revistas latinoamericanas muestran un aumento sostenido del número de publicaciones en el período, destacándose el gran aumento del número de artículos publicados en el Jornal Brasileiro de Pneumologia que alcanza su máximo en 2010 (Figura 3). El aumento del número de publicaciones en esta revista se acompañó de una mayor sumatoria del índice $\mathrm{H}$ en el período (Figura 4).

Además el grupo de revistas de América Latina constituidas por Jornal Brasileiro de Pneumologia, Revista del Instituto Nacional de Enfermedades Respiratorias, México y Revista Chilena de Enfermedades Respiratorias fue comparado con las dos revistas de España que SCImago Journal \& Country Rank identifica en el tema de enfermedades respiratorias: Archivos de Bronconeumología y la Revista Española de Patología ${ }^{7}$. Los dos grupos de revistas fueron comparadas en cuanto a número de artículos citables (Figura 5) y sumatoria de índice H (Figura 6). Se puede observar que las revistas españolas superan en ambos indicadores al conjunto de las tres revistas latinoamericanas.

Al comparar los índices del Journal Citation Report (JRC) del Jornal Brasileiro de Pneumologia que es la revista latinoamericana mejor indexada con Archivos de Bronconeumología, se constata que esta última revista exhibe globalmente mejores índices ${ }^{9}$ (Tabla 4).

Considerando que la ALAT mantiene una

Tabla 3. Índices bibliométricos de seis revistas de enfermedades respiratorias Indexadas en SCImago (información año 2014) ${ }^{7}$

\begin{tabular}{|c|c|c|c|c|c|c|c|c|c|}
\hline Título de la revista & SJR & $\begin{array}{c}\text { Índice } \\
\mathbf{H}\end{array}$ & $\begin{array}{l}\text { Total } \\
\text { Docs } \\
2014\end{array}$ & $\begin{array}{c}\text { Total } \\
\text { Docs } \\
\text { (3 años) }\end{array}$ & $\begin{array}{l}\text { Total } \\
\text { Refs. }\end{array}$ & $\begin{array}{c}\text { Total } \\
\text { citas } \\
\text { (3 años) }\end{array}$ & $\begin{array}{c}\text { Docs } \\
\text { citables } \\
\text { (3 años) }\end{array}$ & $\begin{array}{c}\text { Citas/ } \\
\text { docs } \\
\text { (2 años) }\end{array}$ & $\begin{array}{l}\text { Refs/ } \\
\text { docs }\end{array}$ \\
\hline Archivos Bronco- Neumol & $\begin{array}{c}\mathrm{Q} 2 \\
0,432\end{array}$ & 34 & 245 & 484 & 3.303 & 500 & 303 & 1,82 & 13,48 \\
\hline J Brasileiro Pneumol & $\begin{array}{c}\mathrm{Q3} \\
0,383\end{array}$ & 21 & 100 & 369 & 2.549 & 315 & 276 & 1,04 & 25,49 \\
\hline Revista Portuguesa Neumol & $\begin{array}{c}\text { Q3 } \\
0,294\end{array}$ & 11 & 92 & 212 & 1.531 & 167 & 137 & 1,28 & 16,64 \\
\hline Rev Chilena Enf Respir & $\begin{array}{c}\text { Q3 } \\
0,155\end{array}$ & 6 & 30 & 107 & 450 & 14 & 81 & 0,17 & 15 \\
\hline Rev Inst Nac Enf Respir, Méx & $\begin{array}{c}\mathrm{Q} 4 \\
0,124\end{array}$ & 7 & 33 & 116 & 744 & 7 & 85 & 0,06 & 22,55 \\
\hline R Española Patología & $\begin{array}{c}\mathrm{Q} 4 \\
0,109\end{array}$ & 4 & 110 & 235 & 1.745 & 14 & 193 & 0,06 & 15,86 \\
\hline
\end{tabular}

Docs= documentos; Refs= referencias; Docs citables= artículos originales y de revisión; Q= cuartil. 


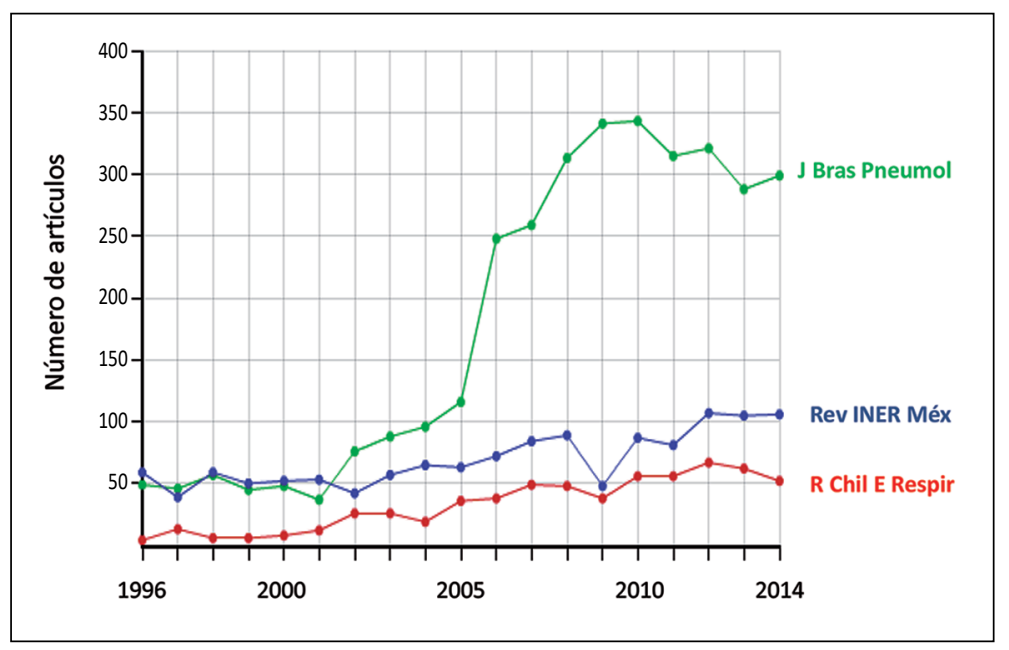

Figura 3. Número de artículos publicados en Jornal Brasileiro de Pneumologia $(\bullet)$, Revista del Instituto Nacional de Enfermedades Respiratorias, México $(\bullet)$ y Revista Chilena de Enfermedades Respiratorias $(\bullet)$. Período 1996-2014. Gráfico adaptado de SCImago ${ }^{7}$.

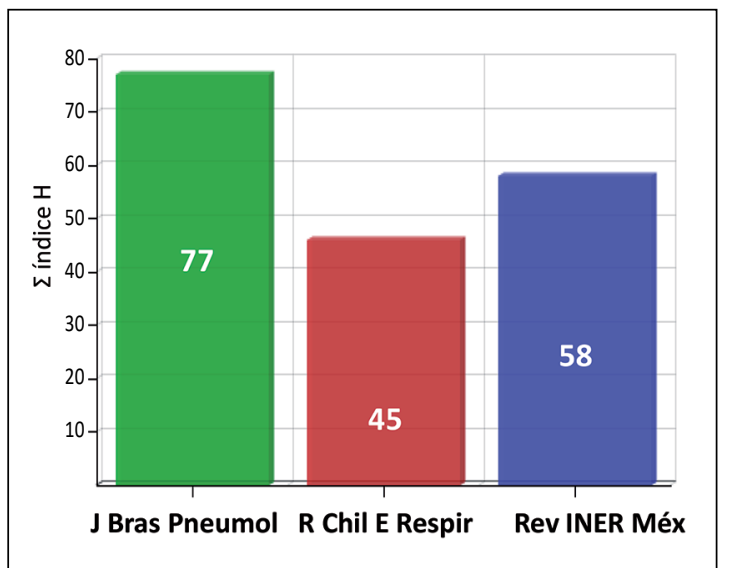

Figura 4. Sumatoria del índice $\mathrm{H}$ en el período 19962014 de Jornal Brasileiro de Pneumologia (घ), Revista del Instituto Nacional de Enfermedades Respiratorias, México ( $\square$ ) y Revista Chilena de Enfermedades Respiratorias (घ). Gráfico adaptado de SCImago ${ }^{7}$. alianza estratégica de publicación con la revista de la Sociedad Española de Neumología y Cirugía del Tórax (SEPAR), Archivos de Bronconeumología, analizamos la contribución de autores de países de América Latina en la publicación de artículos originales, casos clínicos y revisiones publicados en Archivos de Bronconeumología entre 2013 y 2015. Esta contribución de autores de América Latina fluctuó anualmente entre $9 \mathrm{y}$ 12\%: En resumen, de un total de 238 artículos citables publicados en el trienio la gran mayoría de ellos $(76,5 \%)$ fue generado por autores españoles, un $10,9 \%$ tuvo autoría latinoamericana y un $12,6 \%$ correspondió a autores de otros países la mayoría de ellos europeos (Tabla 5).

De lo anteriormente expuesto podemos comentar que las revistas nacionales en esta especialidad son necesarias, porque cumplen un valioso papel docente y de difusión a nivel local en la

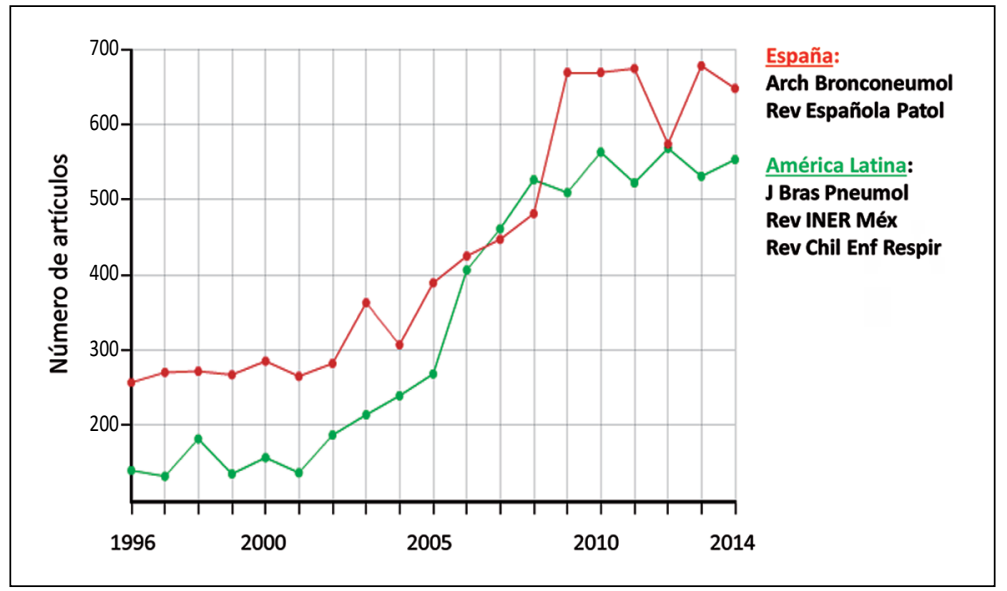

Figura 5. Número de artículos publicados entre 1996 y 2014 en revistas de Enfermedades Respiratorias de América Latina $(\bullet)$ y España $(\bullet)$. Gráfico adaptado de SCImago ${ }^{7}$. 
Tabla 4. Índices Journal Citations Reports (JCR)9 2015:

Jornal Brasileiro de Pneumologia y Archivos de Bronconeumología

\begin{tabular}{|lccccccc|}
\hline Revista & $\begin{array}{c}\text { Total } \\
\text { de citas }\end{array}$ & $\begin{array}{c}\text { Factor de } \\
\text { impacto }\end{array}$ & $\begin{array}{c}\text { Factor de } \\
\text { impacto en } \\
\mathbf{5} \text { años }\end{array}$ & $\begin{array}{c}\text { Índice } \\
\text { inmediatez }\end{array}$ & $\begin{array}{c}\text { Ítemes } \\
\text { citables }\end{array}$ & $\begin{array}{c}\text { Cited } \\
\text { Half-life }\end{array}$ & $\begin{array}{c}\text { Citing } \\
\text { Half- life }\end{array}$ \\
\hline Jornal Brasileiro Pneumol & 1.065 & 1,019 & 1,104 & 0,238 & 63 & 6,1 & 7,1 \\
\hline Archivos Bronconeumología & 1.341 & 1,771 & 1,751 & 0,754 & 69 & 5,3 & 7,2 \\
\hline
\end{tabular}

Total de citas: número de veces que una revista ha sido citada en un año por todas las revistas JCR. Factor de impacto: promedio del $\mathrm{n}^{\circ}$ de veces que los artículos publicados en los 2 últimos años han sido citados en un determinado año por revistas incluidas en JCR. Índice de inmediatez: frecuencia promedio en que son citados los artículos de la revista en el mismo año de publicación. Cited half-life: Media de la edad de los artículos que se citan de una revista. Citing half-life: Media de edad de los artículos citados en una revista.

Tabla 5. Origen de los artículos publicados en Archivos de Bronconeumología 2013-15

\begin{tabular}{|c|c|c|c|c|c|c|c|c|}
\hline \multirow{2}{*}{$\begin{array}{l}\text { Origen } \\
\text { Autores }\end{array}$} & \multicolumn{2}{|c|}{2013} & \multicolumn{2}{|c|}{2014} & \multicolumn{2}{|c|}{2015} & \multicolumn{2}{|c|}{ 2013-15 } \\
\hline & $\mathbf{n}$ & $\%$ & $\mathbf{n}$ & $\%$ & $\mathbf{n}$ & $\%$ & $\mathbf{n}$ & $\%$ \\
\hline España & 62 & 77 & 59 & 80 & 61 & 73 & 182 & 76,5 \\
\hline América Latina & 7 & 9 & 9 & 12 & 10 & 12 & 26 & 10,9 \\
\hline Otros & 11 & 14 & 6 & 8 & 13 & 15 & 30 & 12,6 \\
\hline Total & 80 & 100 & 74 & 100 & 84 & 100 & 238 & 100 \\
\hline
\end{tabular}

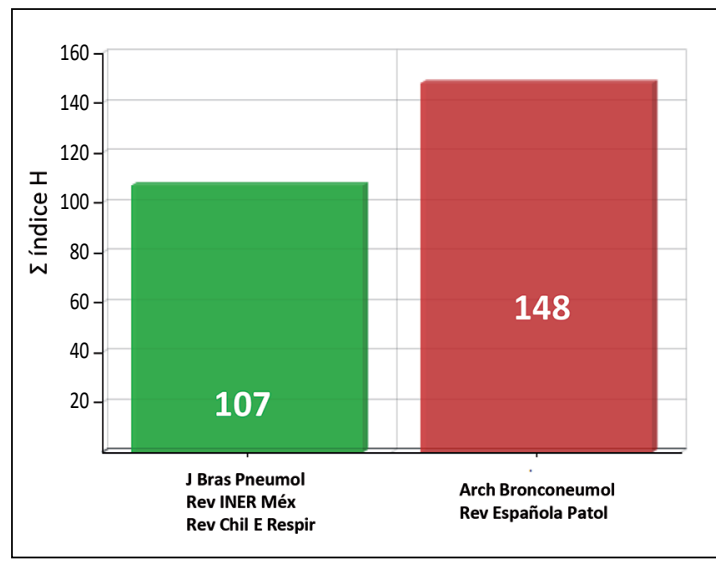

Figura 6. Sumatoria del índice $H$ de revistas de Enfermedades Respiratorias de América Latina ( $\square$ ) y España (匹). Período: 1996-2014. Gráfico adaptado de SCImago ${ }^{7}$.

descripción y cuantificación de las enfermedades prevalentes en un país o región. Por otra parte, si bien la investigación original en esta temática en la región parecería ser comparativamente escasa, la publicación de artículos originales en estas revistas puede constituir un primer paso para llevar a cabo proyectos multicéntricos o de mayor envergadura. Cuando se discutió el tema en el Simposio de la ALAT dedicado a analizar el presente y futuro de las revistas respiratorias en América Latina hubo acuerdo en la idea de aunar esfuerzos para reconsiderar la creación de una revista regional.

\section{Conclusiones}

El análisis bibliométrico realizado en este artículo nos permite plantear las siguientes conclusiones:

a) Las revistas de Enfermedades Respiratorias de América Latina difunden información valiosa para el ámbito nacional que las genera, cumpliendo además una función docente. Sin embargo, su impacto global y sus índices bibliométricos son exiguos.

b) Las revistas de las sociedades nacionales enfrentan en su edición varios problemas comunes y esta situación no se ha modificado sustancialmente en la última década.

c) Archivos de Bronconeumología órgano oficial de la SEPAR y aliado estratégico de la ALAT ha publicado en el último trienio 238 artículos citables (artículos originales y revisiones), $11 \%$ de los cuales han sido generados por autores de América Latina.

d) ALAT debería aunar esfuerzos para lograr editar una Revista de Enfermedades Respiratorias de América Latina y además generar incentivos para estimular la publicación de trabajos originales de la más alta calidad. 


\section{Agradecimientos}

Los autores agradecen al Dr. Francisco Arancibia H., presidente de la Sociedad Chilena de Enfermedades Respiratorias la lectura crítica de este manuscrito.

\section{Bibliografía}

1.- MICHALOPOULUS A, FALAGAS M E. A bibliometric analysis of global research production in respiratory medicine. Chest 2005; 128: 3993-8.

2.- OYARZÚN M. Situación editorial de las revistas latinoamericanas de enfermedades respiratorias. Rev Chil Enferm Respir 2007; 23: 125-31.

3.- PÉREZ-PADILLA R. La inevitable gestación de revistas médicas multinacionales en América Latina. Rev Chil Enferm Respir 2006; 22: 176-80.

4.- OYARZÚN M, RAMÍREZ A, AGÜERO A, BADDINIMARTÍNEZ J A, BERMÚDEZ M, CÁNEVA J O, et al. Estudio situacional de siete revistas latinoamericanas de Neumología. Rev Med Chile 2007; 135: 1072-5.

5.- LITERATURA LATINOAMERICANA EN CIENCIAS DE LA SALUD [INTERNET]. Sao Paulo, Brasil: BIREME-OPS-OMS [citado el 05 de abril de 2016]. Disponible en: http://bases.bireme.br/ cgi-bin/wxislind.exe/iah/online/?IsisScript=iah/iah. $\mathrm{xis} \&$ base $=$ LILACS\&lang $=\mathrm{e}$

6.- SCIENTIFIC ELECTRONIC LIBRARY ONLINE [INTERNET]. Sao Paulo, Brasil: Scientific Electronic Library Online [citado el 05 de abril de 2016]. Disponible en: http://www.scielo.org/php/index.php

7.- SCIMAGO JOURNAL \& COUNTRY RANK [INTERNET]. Madrid, España: SCImago Journal \& Country Rank [citado el 05 de abril de 2016]. Disponible en: http://www.scimagojr.com/

8.- MEDLINE [INTERNET]. Estados Unidos: US National Library of Medicine National Institutes of Health [citado el 02 de mayo de 2016]. Disponible en: http://www. ncbi.nlm.nih.gov/pubmed

9.- JOURNAL CITATION REPORTS [INTERNET]. New York, Estados Unidos: Thomson Reuters [citado el 22 de junio de 2016]. Disponible en: http://admin-apps. webofknowledge.com/JCR/JCR?PointOfEntry=Home $\& \mathrm{SID}=4 \mathrm{CsB} 33 \mathrm{~h} 741 \mathrm{JEgMhuBIt}$

10.- MORAIS A. What we have done so far and what we can achieve (II). Rev Port Pneumol 2015; 21: 51-2 (editorial).

11.- DONATO H. Understanding journal evaluation and strategies to increase impact. Rev Port Pneumol 2016; 22: 67-9 (editorial).

12.- AGUIRRE M, OYARZÚN M. Siete años de evolución de la Revista Chilena de Enfermedades Respiratorias en SciELO: desafíos y oportunidades. Rev Chil Enferm Respir 2009; 25: 5-7. (Editorial).
Correspondencia a:

Dr. Manuel Oyarzún G.

Director, Programa de Fisiopatología, Instituto

de Ciencias Biomédicas, Facultad de Medicina,

Universidad de Chile.

Avda. Salvador 486, Providencia, Santiago, Chile.

Código Postal: 7500922

E mail: moyarzun@med.uchile.cl 